\title{
Avaliação endoscópica das vias aéreas, do perfil de gases, dos eletrólitos e do equilíbrio ácido-base em equinos submetidos ao treinamento de três tambores
}

\author{
[Endoscopic airway, blood gas, electrolytes, and acid-base in equine submitted \\ to three-barrel training] \\ J.P.B. Barbosa ${ }^{1}$, D.S. Denadai ${ }^{2}$, B. Gerardi ${ }^{1}$, M.A. Pereira ${ }^{1}$, A.A. Chaves $^{1}$, L.M.W. Gomide ${ }^{3}$, \\ J.R. Peiró ${ }^{4}$, L.C.N. Mendes ${ }^{4}$ \\ ${ }^{1}$ Aluno de pós-graduação - FMV-Unesp - Campus de Araçatuba, SP \\ ${ }^{2}$ Aluna de graduação - Bolsista de Iniciação Científica - Campus de Araçatuba, SP \\ ${ }^{3}$ Pós-Doutoranda - FMV-Unesp - Campus de Araçatuba, SP \\ ${ }^{4}$ FMV-Unesp - Campus de Araçatuba, SP \\ RESUMO
}

\begin{abstract}
O objetivo deste estudo foi avaliar as vias aéreas por endoscopia, o perfil de gases, de eletrólitos e o equilíbrio ácido-base de equinos da raça Quarto de Milha (QM) antes e após o exercício de três tambores. Dezesseis cavalos, divididos em dois grupos de oito animais, treino regular (TR - cinco vezes) e treino esporádico (TE duas vezes por semana), foram utilizados no experimento. A endoscopia foi realizada antes e 90 min após o exercício. Foram realizadas duas coletas de sangue por punção da artéria facial transversa antes do aquecimento (M0) e imediatamente após o exercício (M1), e as leituras foram realizadas com o I-Stat ${ }^{\circledR}$. Foram determinadas $\mathrm{PCO}_{2}, \mathrm{PO}_{2}, \mathrm{SO}_{2}, \mathrm{pH}, \mathrm{BE}, \mathrm{HCO}_{3}$ e $\mathrm{TCO}_{2}, \mathrm{Na}^{+}, \mathrm{K}^{+}$, iCa, glicose, $\mathrm{Hg}$ e Ht. Foi utilizada ANOVA com medidas repetidas no tempo para comparações dos grupos e dos momentos $(\mathrm{P}<0,05)$. A HFL acometeu em grau leve (12) os animais de ambos os grupos, com $8 / 8(100 \%)$ no TR e $5 / 8(62,5 \%)$ no TE. Observou-se presença de secreção serosa na traqueia em 4/8 (50\%) para ambos os grupos. A HPIE acometeu em grau leve (1-2) os animais de ambos os grupos, com 4/8 (50\%) no grupo TR e 3/8 (37,5\%) no grupo TE. Os animais com grau 2 de HPIE apresentaram leve hipoxemia e hipercapnia pós-exercício. $\mathrm{SO}_{2}, \mathrm{PCO}_{2}, \mathrm{pH}, \mathrm{HCO}_{3}, \mathrm{BE}, \mathrm{K}$ e iCa ${ }^{+}$não diferiram estatisticamente $(\mathrm{P}>0,05)$ entre os grupos. Os cavalos da raça $\mathrm{QM}$ submetidos ao exercício de TT apresentam HPIE e acidose metabólica.
\end{abstract}

Palavras-chave: equino, hemogasometria, hemorragia pulmonar, exercício

\begin{abstract}
The aim of this study was to evaluate the airways by endoscopy, gas profile, electrolytes and acid-base balance of horses in the Quarter Horse (QM) race before and after training three drums. Sixteen horses divided into two groups of 8 animals, with regular training ( $R T$ - 5 times a week) and sporadic training (ET - 2 times a week) were used in the experiment. Endoscopy was performed before and 90 min after training. Two blood samples were taken by puncturing the transverse facial artery before heating (M0) and immediately after training (M1), and the readings were performed with the I-Stat ${ }^{\circledR}$. PCO2, PO2, SO2, pH, BE, HCO3 and TCO2, $\mathrm{Na}^{+}, \mathrm{K}^{+}$, iCa, Glucose, $\mathrm{Hg}$, and Ht were determined. ANOVA was used with repeated measures over time for comparisons of groups and time $(P<0.05)$. The HFL befell was mild $(1-2)$, the animals from both groups with 8/8 (100\%) in the TR and 5/8 (62.5\%) for the TE. There was presence of serous trachea 4/8 (50\%) for both groups. The EIPH befell was mild (1-2) in the animals from both groups with 4/8 (50\%) in the RT group and 3/8 (37.5\%) for the TE group. Animals with grade 2 EIPH had mild hypoxemia and hypercapnia after exercise. The SO2, $\mathrm{PCO} 2, \mathrm{pH}, \mathrm{HCO}, \mathrm{BE}, \mathrm{K}$, and iCa did not differ $\mathrm{P}<0.05$ between groups. QM race submitted to TT exercise have EIPH and metabolic acidosis.
\end{abstract}

Keywords: equine, blood gas, pulmonary hemorrhage, exercise

Recebido em 1 de setembro de 2015

Aceito em 1 de março de 2016

*Autor para correspondência (corresponding author)

E-mail:daniela denadai@hotmail.com

Apoio: FAPESP n.2014/23012-7 - Unesp-Araçatuba 


\section{INTRODUÇÃO}

A competição de três tambores (TT) requer do conjunto (cavalo e cavaleiro) habilidade e velocidade para percorrer, no menor tempo, um trajeto triangular contornando três tambores. A raça mais utilizada é a Quarto de Milha, devido a sua capacidade atlética e à maior aceleração entre os equinos (Silva et al., 2013).

A avaliação endoscópica, antes e após o exercício, é uma ferramenta útil na clínica médica e esportiva dos equinos, auxiliando no diagnóstico de doenças do sistema respiratório. Possibilita avaliar tanto as vias aéreas superiores quanto as inferiores, identificando afecções como a hiperplasia folicular linfoide (HFL) e a hemorragia pulmonar induzida por exercício (HPIE) (Hinchcliff et al., 2015). A gasometria arterial, associada ao exame endoscópico, é utilizada na identificação do comportamento respiratório de cavalos em situação de treino e em competições. O resultado instantâneo da gasometria arterial possibilita avaliar a eficácia das trocas gasosas e a correlação desta com sinais observados na endoscopia (Sanchez et al., 2005).

Nos exercícios de treinamento de TT, o cavalo precisa atingir alta velocidade em curtíssimo espaço de tempo. Necessita de alta produção de energia e apropriado consumo para suprir a demanda da intensa contração muscular. Essa energia é gerada via metabolismo anaeróbio (glicólise). Metabolitos são produzidos por essa via (ácido láctico e lactato), na qual são dissociados, gerando prótons hidrogênio $(\mathrm{H}+)$, com consequente diminuição do $\mathrm{pH}$ sanguíneo e acidose metabólica (Marlin e Nankervis, 2002).

Diante do exposto, a hipótese proposta pelo estudo foi que o exercício de TT provoca hemorragia pulmonar, alterando a troca gasosa, e desequilíbrio de eletrólitos e ácido-base. Logo, o objetivo do presente estudo foi avaliar as vias aéreas, o perfil de gases, eletrólitos, o equilíbrio ácido-base e a velocidade máxima de equinos submetidos ao treinamento de três tambores.

\section{MATERIAL E MÉTODOS}

Foram avaliados 16 equinos da raça QM durante exercício para a modalidade TT, provenientes da região de Araçatuba-SP. Foram quatro machos castrados e 12 fêmeas, com média de idade e peso de $6 \pm 3$ anos e $436 \pm 36 \mathrm{~kg}$, respectivamente, distribuídos em dois grupos compostos por oito animais cada, divididos por nível de treinamento regular (TR $-5 \mathrm{x}$ por semana) e esporádico (TE - $2 x$ por semana). Os animais eram mantidos em baias, alimentados com feno, concentrado e água ad libidium, vermifugados e vacinados contra influenza, rinopneumonite, encefalomielite e tétano (Lexington $8^{\circledR}$ ).

Realizou-se investigação da história clínica, endoscopia do trato respiratório e exame de claudicação. Eram considerados inaptos a participar do experimento os animais que apresentaram sintomas como secreção nasal, tosse, anormalidades anatômicas ou afecções respiratórias que poderiam comprometer a passagem normal do ar, aumento de volume ou dor à palpação em tendões e ligamentos dos membros ou claudicação.

Para a realização segura do exame endoscópico, foi necessário que uma pessoa direcionasse $o$ aparelho, outra controlasse as funções dele, além de um ajudante na contenção física dos animais. Para iniciar o exame endoscópico, o aparelho foi direcionado contra o meato ventral da cavidade nasal. As primeiras porções examinadas foram as regiões faríngeas e laríngeas. Na região faríngea, observaram-se estruturas como o recesso faríngeo, a abertura das bolsas guturais e os folículos linfoides. $\mathrm{Na}$ região laríngea, observaram-se principalmente as cartilagens da epiglote e aritenoides. Os processos corniculados das cartilagens aritenoides foram examinados quanto às anormalidades (estruturais, ulcerações, tecido de granulação e espessura) e, ainda, sua movimentação durante a inspiração e a expiração. Posteriormente, o endoscópio foi inserido na traqueia para a determinação de possíveis secreções mucopurulentas, sangue, coloração e reatividade traqueal à passagem do endoscópio. A endoscopia foi realizada previamente e 90min após o exercício, com uso de aparelho endoscópio flexível Endoscópio Storz ${ }^{\circledR}$, GE, com $1,70 \mathrm{~m}$ de comp., $12 \mathrm{~mm}$ de diâmetro e uma fonte de luz fria de 150watt (Michelotto, 2007; Hinchcliff et al., 2015).

A presença de folículos linfoides na faringe foi graduada segundo Raker e Bowles (1978), adotando-se a graduação de I a IV, e para a 
graduação da hemorragia pulmonar induzida por exercício, foi utilizada a classificação de Hinchcliff et al. (2004) descrita nas Tab. 1 e 2, respectivamente.

Os parâmetros avaliados no exame físico incluíram: frequência cardíaca (FC), frequência respiratória (FR), hidratação, coloração de mucosas, temperatura retal e presença de secreção nasal ou epistaxe. Os equinos foram avaliados 30 minutos antes (M0), logo após (1 a 3 min - M1) e depois de 90 min (M2) do exercício. Amostras de sangue $(0,6 \mathrm{~mL})$ foram coletadas por punção da artéria facial transversa com seringa de $1 \mathrm{~mL}$ para gasometria com heparina de lítio $\mathrm{BD}{ }^{\circledR}$ (A-LINE) e agulha
$13 \times 4,5$, antes do aquecimento (M0) e até $3 \mathrm{~min}$ após o término do exercício (M1) (Silva et al., 2012). As leituras das amostras foram realizadas no local de treinamento, com uso do aparelho de hemogasometria portátil I-Stat ${ }^{\circledR}$ Portable Clinical Analyzer, Abbott Laboratories, Abbott Park, Illinois, EUA, onde foram determinadas a pressão arterial de dióxido de carbono $\left(\mathrm{PaCO}_{2}\right)$, a pressão arterial de oxigênio $\left(\mathrm{PO}_{2}\right)$, a saturação de oxigênio $\left(\mathrm{sO}_{2}\right)$, o logaritmo negativo da atividade de íons hidrogênio $(\mathrm{pH})$, o hematócrito $(\mathrm{Ht})$, as concentrações de bases tituláveis $(\mathrm{BE})$, o íon bicarbonato $\left(\mathrm{HCO}_{3}\right)$, o dióxido de carbono total $\left(\mathrm{TCO}_{2}\right)$, o íon sódio $\left(\mathrm{Na}^{+}\right)$, o íon potássio $\left(\mathrm{K}^{+}\right)$, o cálcio ionizado (Cai), a glicose (Glu) e a hemoglobina total $(\mathrm{Hb})$.

Tabela 1. Graduação da presença de folículos linfoides na faringe de equinos descrita por Raker e Bowles (1978)

\begin{tabular}{c|l}
\hline Grau & \multicolumn{1}{|c}{ Graduação de folículos linfoides } \\
\hline 1 & Faringe com poucos folículos claros na parede dorsal \\
\hline 2 & $\begin{array}{l}\text { Presença de numerosos pequenos folículos juntamente à hiperemia, cobrindo as } \\
\text { superfícies dorsal e lateral da faringe }\end{array}$ \\
\hline 3 & $\begin{array}{l}\text { Presença de grandes grupos de folículos hiperêmicos cobrindo as superfícies dorsal e } \\
\text { lateral da faringe }\end{array}$ \\
\hline 4 & Presença de folículos grandes, edematosos e hiperêmicos por toda a mucosa faringeana \\
\hline
\end{tabular}

Tabela 2. Sistema de classificação da hemorragia pulmonar induzida por exercício em cavalos por endoscopia das vias aéreas inferiores (adaptado de Hinchcliff et al., 2004)

\begin{tabular}{c|l}
\hline Grau & \multicolumn{1}{c}{ Sistema de classificação da HPIE por endoscopia } \\
\hline 0 & Ausência de sangue na faringe, laringe, brônquios principais visíveis desde a carina \\
\hline 1 & $\begin{array}{l}\text { Presença de uma ou mais manchas de sangue ou }<2 \text { filamentos de sangue pequenos }(<1 / 4 \text { de } \\
\text { comprimento da traqueia) e estreitos }(<10 \% \text { da superfície) na traqueia ou nos brônquios } \\
\text { principais }\end{array}$ \\
\hline 2 & $\begin{array}{l}\text { Um longo filamento de sangue (em mais da metade do comprimento da traqueia) ou }>2 \\
\text { filamentos curtos que ocupam menos de } 1 / 3 \text { da circunferência traqueal }\end{array}$ \\
\hline 3 & $\begin{array}{l}\text { Filamentos de sangue múltiplos de distintos, que cobrem mais de } 1 / 3 \text { da circunferência } \\
\text { traqueal. Sem acúmulo de sangue na traqueia torácica }\end{array}$ \\
\hline 4 & $\begin{array}{l}\text { Filamentos de sangue múltiplos que se aglutinam, cobrindo }>90 \% \text { da superfície traqueal. Com } \\
\text { acúmulo de sangue na traqueia torácica }\end{array}$ \\
\hline HPIE - hemorragia pulmonar induzida por exercício.
\end{tabular}

Os animais realizaram exercício de aquecimento ao passo, trote e galope, além de alongamentos e flexão de nuca. Ao concluírem o exercício, realizaram uma passada na pista três tambores, com medições semelhantes às provas oficiais da Associação Brasileira de Criadores de Cavalos Quarto de Milha (ABQM). Com o uso do sensor GPS Polar $\mathrm{G} 1{ }^{\circledR}$ no antebraço direito do cavaleiro e do relógio receptor de dados no pulso direito, foi determinada a velocidade máxima $(\mathrm{km} / \mathrm{h})$. Os animais foram treinados pelo mesmo cavaleiro.
Para aferição e coleta das medidas de temperatura e umidade ambiente, foi utilizado um termo-higrômetro modelo Klimalogg Pro ${ }^{\circledR}$ 3030.39.0.00.

Utilizou-se análise de variância com medidas repetidas no tempo para comparações dos grupos e dos momentos. O teste de correlação de Spearman foi utilizado para as variáveis HPIE e velocidade máxima, além do teste de MannWhitney para HFL e HPIE $(\mathrm{P}<0,05)$. 
Este trabalho de pesquisa foi aprovado pelo Comitê de Ética em Experimentação Animal da Unesp - Araçatuba, sob o número de registro 20/12-01985.

\section{RESULTADOS E DISCUSSÃO}

As frequências das afecções apresentadas pelos animais antes e após o exercício estão descritas na Tab. 3. No exame endoscópico realizado antes do exercício, foi observada ocorrência de HFL grau $1 \mathrm{em} 25 \%$ dos animais. Não obstante, após o exercício, observou-se ocorrência de 100\% para os animais do grupo TR e de $62,5 \%$ para os do TE. Segundo Holcombe (2006), a exposição constante e cumulativa à poeira orgânica e a proximidade da faringe ao ambiente externo predispõem à inflamação.

Constatou-se velocidade máxima atingida pelos animais de $44 \mathrm{~km} / \mathrm{h}$. Não houve diferença estatística entre as velocidades dos grupos TR e TE, com médias de $39,9 \mathrm{~km} / \mathrm{h} \pm 3,10$ e $37,9 \mathrm{~km} / \mathrm{h} \pm 2,16$, respectivamente. Os animais que apresentaram HPIE atingiram média de velocidade superior a $36 \mathrm{~km} / \mathrm{h}$, com exceção para o animal 1 do grupo TR. Em estudo realizado por Goetz et al. (2001), os animais foram submetidos a $14 \mathrm{~m} / \mathrm{s} \quad(50 \mathrm{~km} / \mathrm{h})$ em esteira de alta performance, e todos apresentaram algum grau de HPIE, mas não foi realizada a correlação estatística entre as variáveis. No entanto, neste experimento, foi observada a correlação $(\mathrm{r}=0,75)$ entre HPIE e velocidade no grupo TE. Thomas et al. (1983) relataram que cavalos PSI de corrida submetidos a exercício intenso apresentam aumento linear do débito cardíaco conforme incremento da velocidade, com aumento da pressão arterial e, consequentemente, em capilares pulmonares (Langsetmo et al., 2000). Os resultados sugerem que os animais do grupo TE não estão adaptados e condicionados ao treinamento, logo não suportariam altas velocidades e consequente aumento da pressão arterial, estando predispostos a falhas nos capilares pulmonares, corroborando a teoria (HPIE) mais aceita entre os pesquisadores.

Tabela 3. Frequência da hemorragia pulmonar induzida por exercício (HPIE) e da hiperplasia folicular linfoide (HFL) em equinos da raça Quarto de Milha, observados por exame endoscópico, após treinamento de três tambores

\begin{tabular}{|c|c|c|c|c|c|c|}
\hline \multirow{2}{*}{ Variável } & \multirow{2}{*}{ Escore } & \multicolumn{2}{|c|}{ TR $(n=8)$} & \multicolumn{2}{|c|}{$\mathrm{TE}(\mathrm{n}=8)$} & \multirow{2}{*}{$\mathrm{P}^{1}$} \\
\hline & & $\mathrm{N}$ & $\%$ & $\mathrm{~N}$ & $\%$ & \\
\hline \multirow{3}{*}{ HFL } & 0 & 0 & 0 & 4 & 50,0 & \multirow{3}{*}{0,0459} \\
\hline & 1 & 5 & 62,5 & 3 & 37,5 & \\
\hline & 2 & 3 & 37,5 & 1 & 12,5 & \\
\hline \multirow{3}{*}{ HPIE } & 0 & 3 & 37,5 & 5 & 62,5 & \multirow{3}{*}{0,0491} \\
\hline & 1 & 4 & 50,0 & 2 & 25,0 & \\
\hline & 2 & 1 & 12.5 & 1 & 12,5 & \\
\hline
\end{tabular}

TR - grupo treino regular; TE - grupo treino esporádico; $\mathrm{n}$ - número de animais. ${ }^{1}$ Teste de MannWhitney, $\mathrm{P}<0,05$.

Os valores obtidos de $\mathrm{PaO} 2, \mathrm{PaCO} 2, \mathrm{SaO} 2$, logaritmo negativo da atividade de íons hidrogênio $(\mathrm{pH})$, bicarbonato (HCO-3), total de dióxido de carbono (TCO2) e bases tituláveis (BE) estão relacionados na Tab. 4. O aumento de $\mathrm{PaO} 2$ e a diminuição de $\mathrm{PaCO} 2$ em ambos os grupos se devem à hiperventilação compensatória à diminuição do $\mathrm{pH}$ (Hinchcliff et al., 2009). Durante o exercício máximo, há aumento significativo do débito cardíaco, aumentando o volume de sangue presente nos capilares dos alvéolos (Poole e Erickson, 2004); além disso, ocorre aumento da velocidade do fluxo sanguíneo na artéria pulmonar.
Consequentemente, o tempo de trânsito dos eritrócitos pelos capilares diminui e alguns eritrócitos que passam pelos capilares alveolares não conseguem captar as moléculas de $\mathrm{O} 2$, sendo esta, também, causa importante no desenvolvimento da hipoxemia (Wilkins et al., 2001).

Os animais 2 e 11 dos grupos TR e TE apresentaram HPIE grau 2 após exercício, com valores inferiores de $\mathrm{PO}_{2}(89 \mathrm{mmHg}-90 \mathrm{mmHg})$, e superiores de $\mathrm{PCO}_{2}(50,4 \mathrm{mmHg}-49,9 \mathrm{mmHg})$, respectivamente. Isso demonstra possível desenvolvimento de hipoxemia e hipercapnia 
durante o exercício. O animal 1 do grupo TR apresentou hipoxemia e hipercapnia após exercício, com valores de $\mathrm{PO}_{2} 83 \mathrm{mmHg}$ e de $\mathrm{PCO}_{2} 50,4 \mathrm{mmHg}$. Este animal apresentou, além de HPIE e HFL, acúmulo de secreção seromucosa na traqueia, indicando provável acometimento por obstrução recorrente das vias aéreas (ORVA) ou doença inflamatória das vias aéreas (DIVA), visto que, após o experimento, ele apresentou intolerância ao exercício, com dispneia, tosse e acúmulo de secreção na traqueia. Uma hipótese proposta é que qualquer doença que venha a aumentar as pressões em vasos pulmonares ou a aumentar a magnitude das pressões no pulmão durante a inspiração, como a ORVA, promoveria aumento no grau de HPIE (Marlin, 2009).

Tabela 4. Médias \pm desvio-padrão obtidas para as concentrações da pressão arterial de oxigênio $(\mathrm{PaO} 2)$, pressão arterial de gás carbônico ( $\mathrm{PaCO} 2)$, saturação de oxigênio $(\mathrm{SaO} 2)$, logaritmo negativo da atividade de íons hidrogênio $(\mathrm{pH})$, bicarbonato (HCO-3), total de dióxido de carbono ( $\mathrm{TCO} 2)$ e bases tituláveis (BE) em equinos da raça Quarto de Milha, antes e após o treinamento de três tambores

\begin{tabular}{|c|c|c|c|c|c|c|c|c|c|}
\hline \multirow{2}{*}{ Variável } & \multirow{2}{*}{ Momento } & \multicolumn{4}{|c|}{ TR $n=8$} & \multicolumn{4}{|c|}{ TE $n=8$} \\
\hline & & \multicolumn{4}{|c|}{$\overline{\mathbf{x}} \pm \mathrm{S}$} & \multicolumn{4}{|c|}{$\overline{\mathbf{x}} \pm \mathrm{S}$} \\
\hline \multirow{2}{*}{$\mathrm{PaO} 2$ (mmHg) } & M0 & 99,50 & \pm & 3,16 & $\mathrm{a}$ & 103,00 & \pm & 2,33 & $\mathrm{a}$ \\
\hline & M1 & 106,50 & \pm & 13,04 & $\mathrm{~b}$ & 114,50 & \pm & 11,15 & $\mathrm{~b}$ \\
\hline \multirow{2}{*}{ PaCO2 (mmHg) } & M0 & 41,70 & \pm & 3,19 & $\mathrm{a}$ & 37,9 & \pm & 4,22 & $\mathrm{a}$ \\
\hline & M1 & 30,80 & \pm & 10,69 & $\mathrm{~b}$ & 28,1 & \pm & 6,09 & $\mathrm{~b}$ \\
\hline \multirow{2}{*}{$\mathrm{SaO} 2(\%)$} & M0 & 95,25 & \pm & 2,71 & $\mathrm{a}$ & 97,25 & \pm & 1,16 & $\mathrm{a}$ \\
\hline & M1 & 97,75 & \pm & 4,02 & $\mathrm{~b}$ & 98,25 & \pm & 2,52 & $\mathrm{~b}$ \\
\hline \multirow{2}{*}{$\mathrm{pH}$} & M0 & 7,47 & \pm & 0,03 & $\mathrm{a}$ & 7,44 & \pm & 0,07 & $\mathrm{a}$ \\
\hline & M1 & 7,25 & \pm & 0,04 & $\mathrm{~b}$ & 7,24 & \pm & 0,06 & $\mathrm{~b}$ \\
\hline \multirow{2}{*}{$\mathrm{HCO}-3$ (mmoL) } & M0 & 28,01 & \pm & 0,89 & $\mathrm{~b}$ & 22,20 & \pm & 4,66 & $\mathrm{~b}$ \\
\hline & M1 & 11,6 & \pm & 2,72 & $\mathrm{a}$ & 11,20 & \pm & 2,41 & $\mathrm{a}$ \\
\hline \multirow{2}{*}{$\mathrm{TCO} 2(\mathrm{mmo} / \mathrm{L})$} & M0 & 30,25 & \pm & 1,28 & $\mathrm{~b}$ & 29 & \pm & 1,06 & $\mathrm{~b}$ \\
\hline & M1 & 12,75 & \pm & 3,27 & $\mathrm{a}$ & 13 & \pm & 2,39 & $\mathrm{a}$ \\
\hline \multirow{2}{*}{$\mathrm{BE}(\mathrm{mEq} / \mathrm{L})$} & M0 & 3,88 & \pm & 1,35 & $\mathrm{a}$ & 4,38 & \pm & 0,74 & $\mathrm{a}$ \\
\hline & M1 & $-16,38$ & \pm & 3,42 & $\mathrm{~b}$ & $-16,35$ & \pm & 2,91 & $\mathrm{~b}$ \\
\hline
\end{tabular}

TR - grupo treino regular; TE - grupo treino esporádico; $\mathrm{n}$ - número de animais. Letras maiúsculas destacam diferenças entre colunas, enquanto letras minúsculas indicam diferenças entre linhas. Médias seguidas de letras diferentes diferem entre si ao nível de $5 \%$ de probabilidade $(\mathrm{P} \leq 0,05)$.

Os resultados observados evidenciam acidose metabólica pós-exercício, corroborando Silva et al. (2013), que relatam também a diminuição do $\mathrm{HCO}_{3}{ }^{-}$, com a finalidade de tamponamento. Essa diminuição do $\mathrm{pH}, \mathrm{HCO}_{3}$ e $\mathrm{BE}$ observada no exercício máximo é resultado da difusão do ácido láctico produzido pelas células musculares para a circulação sanguínea, no qual o requisito energético das células musculares é mantido predominantemente pelo metabolismo anaeróbio da glicose, resultando em desenvolvimento de acidemia e consequente acidose metabólica (Marlin e Nankervis, 2002).

Os eletrólitos sanguíneos $\mathrm{Na}^{+}$e $\mathrm{K}^{+}$apresentaram diminuição significativa após o exercício, como descrito na Tab. 5. Geralmente não ocorrem alterações intensas no equilíbrio eletrolítico durante os exercícios de intensidade máxima, devido ao período de curta duração (Silva et al.,
2009). Essa alteração pode estar relacionada à temperatura local nos dias de treinamento, visto que, apesar da diferença entre os momentos e os grupos, a concentração de $\mathrm{Na}^{+}$se manteve dentro dos padrões normais para a espécie equina, 132 a $146 \mathrm{mEq} / \mathrm{L}$ (Hinchcliff et al., 2002). A diminuição do $\mathrm{K}^{+}$está relacionada ao incremento da atividade da bomba de $\mathrm{Na}+/ \mathrm{K}+$ ATPase, com aumento da recaptação do $\mathrm{K}^{+}$e consequente diminuição das concentrações plasmáticas deste cátion (Di Fillipo et al., 2009).

As concentrações de glicose, hemoglobina e hematócrito estão descritas na Tab. 6. A hiperglicemia observada, principalmente no grupo TR, evidencia o uso de metabolismo anaeróbio, como observado por Ferraz et al. (2008), ao utilizarem teste incremental, devido à ação de catecolaminas e do glucagon, que atuam no fígado promovendo a glicogenólise. 
Tabela 5. Médias \pm desvio-padrão obtidos para as concentrações de sódio $\left(\mathrm{Na}^{+}\right)$, potássio $\left(\mathrm{K}^{+}\right)$e cálcio ionizado (iCa) dos equinos da raça Quarto de Milha, antes e após o treinamento de três tambores

\begin{tabular}{|c|c|c|c|c|c|c|c|c|c|}
\hline \multirow{2}{*}{ Variável } & \multirow{2}{*}{ Momento } & \multicolumn{4}{|c|}{ TR $n=8$} & \multicolumn{4}{|c|}{$\mathrm{TE} n=8$} \\
\hline & & \multicolumn{4}{|c|}{$\bar{x} \pm \mathrm{S}$} & \multicolumn{4}{|c|}{$\bar{x} \pm \mathrm{S}$} \\
\hline \multirow{2}{*}{$\mathrm{Na}^{+}(\mathrm{mmo} / \mathrm{L})$} & $\mathrm{M}_{0}$ & 137,10 & \pm & 1,51 & $\mathrm{a}$ & 135,13 & \pm & 2,03 & $\mathrm{~b}$ \\
\hline & $\mathrm{M}_{1}$ & 135,13 & \pm & 2,03 & $\mathrm{Ab}$ & 133,10 & \pm & 2,00 & $\mathrm{Ba}$ \\
\hline \multirow{2}{*}{$\mathrm{K}^{+}(\mathrm{mmo} / \mathrm{L})$} & $\mathrm{M}_{0}$ & 3,99 & \pm & 0,36 & $\mathrm{a}$ & 4,03 & \pm & 1,19 & $\mathrm{a}$ \\
\hline & $\mathrm{M}_{1}$ & 3,65 & \pm & 0,17 & $\mathrm{~b}$ & 3,51 & \pm & 0,90 & $\mathrm{~b}$ \\
\hline \multirow{2}{*}{$\mathrm{iCa}(\mathrm{mmo} / \mathrm{L})$} & $\mathrm{M}_{0}$ & 1,52 & \pm & 0,16 & $\mathrm{a}$ & 1,35 & \pm & 0,13 & $\mathrm{~b}$ \\
\hline & $\mathrm{M}_{1}$ & 1,51 & \pm & 0,20 & $\mathrm{a}$ & 1,31 & \pm & 0,06 & $\mathrm{~b}$ \\
\hline
\end{tabular}

TR - grupo treino regular; TE - grupo treino esporádico; $\mathrm{n}$ - número de animais. Letras maiúsculas destacam diferenças entre colunas, enquanto letras minúsculas indicam diferenças entre linhas. Médias seguidas de letras diferentes diferem entre si ao nível de $5 \%$ de probabilidade $(\mathrm{P} \leq 0,05)$.

Tabela 6. Médias \pm desvio-padrão obtidos para as concentrações de glicose (Gli) hemoglobina (Hb) e hematócrito (Ht) em equinos da raça Quarto de Milha, antes e após o treinamento de três tambores

\begin{tabular}{|c|c|c|c|c|c|c|c|c|c|}
\hline \multirow{2}{*}{ Variável } & \multirow{2}{*}{ Momento } & \multicolumn{4}{|c|}{ TR $n=8$} & \multicolumn{4}{|c|}{ TE $n=8$} \\
\hline & & \multicolumn{4}{|c|}{$\bar{x} \pm \mathrm{S}$} & \multicolumn{4}{|c|}{$\overline{\boldsymbol{x}} \pm \mathrm{S}$} \\
\hline \multirow{2}{*}{ Gli (mmo/L) } & $\mathrm{M}_{0}$ & 106,00 & \pm & 6,30 & $\mathrm{a}$ & 111,13 & \pm & 10,84 & $\mathrm{~b}$ \\
\hline & $\mathrm{M}_{1}$ & 116,75 & \pm & 16,27 & $\mathrm{~b}$ & 115,25 & \pm & 15,30 & $\mathrm{a}$ \\
\hline \multirow{2}{*}{$\mathrm{Hb}(\mathrm{g} / \mathrm{dL})$} & $\mathrm{M}_{0}$ & 11,63 & \pm & 2,91 & $\mathrm{a}$ & 11,56 & \pm & 1,44 & $\mathrm{~b}$ \\
\hline & $\mathrm{M}_{1}$ & 15,15 & \pm & 2,79 & $\mathrm{~b}$ & 15,90 & \pm & 1,50 & $\mathrm{a}$ \\
\hline \multirow{2}{*}{ Ht $(\%)$} & $\mathrm{M}_{0}$ & 34.90 & \pm & 5,40 & $\mathrm{a}$ & 34,60 & \pm & 4,13 & $\mathrm{a}$ \\
\hline & $\mathrm{M}_{1}$ & 46,10 & \pm & 5,89 & $\mathrm{~b}$ & 46,80 & \pm & 3,28 & $\mathrm{~b}$ \\
\hline
\end{tabular}

TR - grupo treino regular; TE - grupo treino esporádico; $\mathrm{n}$ - número de animais. Letras maiúsculas destacam diferenças entre colunas, enquanto letras minúsculas indicam diferenças entre linhas. Médias seguidas de letras diferentes diferem entre si ao nível de $5 \%$ de probabilidade $(\mathrm{P} \leq 0,05)$.

Ambos os grupos apresentaram diferença significativa nas concentrações de hematócrito e hemoglobina pós-exercício. Esse aumento é decorrente da esplenocontração, fenômeno fisiológico considerado como um dos fatores determinantes do aumento do volume sanguíneo durante o exercício, mediada pela ação de catecolaminas, pelo incremento do débito cardíaco (Ferraz et al., 2009) e pela íntima ligação entre o oxigênio e a hemoglobina no transporte deste para os tecidos (Poole e Erickson, 2004).

\section{CONCLUSÃO}

Os equinos submetidos ao exercício de três tambores proposto no presente estudo desenvolvem HPIE e acidose metabólica. No entanto, o exercício não foi significativamente suficiente para comprometer a troca gasosa e alterar o equilíbrio eletrolítico. Complementarmente, a correlação positiva entre velocidade e HPIE nos animais de treino esporádico evidencia a importância do acompanhamento endoscópico do cavalo de TT visando à prevenção, ao desempenho atlético e a uma longa vida desportiva.

\section{REFERÊNCIAS}

FERRAZ, G.C.; DANGELIS, F.H.F.; TEIXEIRA-NETO, A.R. et al. Blood lactate threshold reflects glucose responses in horses submitted to incremental exercise test. Arq. Bras. Med. Vet. Zootec., v.60, p.256-259, 2008.

FERRAZ, G.C.; TEIXEIRA-NETO, A.R.; D'ANGELIS, F.H.F. et al. Alterações hematológicas e cardíacas em cavalos Árabes submetidos ao teste de esforço crescente em esteira rolante. Bras. J. Vet. Res. Anim. Sci., v.46, p.431-437, 2009.

FILLIPO, P.A.; SANTANA, A.E.; PEREIRA, G.T. Equilíbrio ácido-base e hidroeletrolítico em equinos com cólica. Ciênc. Rural, v.38, p.10031009, 2009. 
GOETZ, T.E.; MANOHAR, M.; HASSAN, A.S. Nasal strips do not affect pulmonary gas exchange, anaerobic metabolism, or EIPH in exercising Thoroughbreds. J. Appl. Physiol., v.90, p.2378-85, 2001.

HINCHCLIFF K.W.; KANEPS, A.J.; GEOR, R.J. (Ed.). Equine sports medicine and surgery. Philadelphia: Saunders, 2004. 633-656p.

HINCHCLIFF, K.W.; COUETIL, L.L.; MORLEY, P.S. et al. Exercise induced pulmonary hemorrhage in horse: American college of veterinary internal medicine consensus statement. J. Vet. Intern. Med., v.29, p.743-758, 2015.

HINCHCLIFF, K.W.; LAUDERDALE, M.A.; DUTSON, J. et al. High intensity exercise conditioning increases accumulated oxygen deficit off horses. Equine Vet. J., v.34, p.9-16, 2002.

HINCHCLIFF, K.W.; MORLEY, P.S.; GUTHRIE, A.J. Efficacy of furosemide for prevention of exercise-induced pulmonary hemorrhage in Thoroughbred racehorses. J. Am. Vet. Med. Assoc., v.235, p.76-82, 2009.

HOLCOMBE, S. Upper airway anatomy and physiology gone wrong: how do we diagnose the problem and what can we fix? In: ANNUAL RESORT SYMPOSIUM OF THE AMERICAN ASSOCIATION OF EQUINE PRACTITIONERS, 2006, Rome. Anais... Rome: [AAEP], 2006. p.10-27.

LANGSETMO, I.; FEDDE M.R.; MEYER T.S. Relationship of pulmonary arterial pressure to pulmonary haemorrhage in exercising horses. Equine Vet. J., v.32, p.379-384, 2000.

MARLIN, D.; NARKERVIS, K.J. (Eds.). Equine exercise physiology. Oxford: Wiley BlackwelL, 2002. 304p.

MARLIN, D.J. Exercise-induced pulmonary haemorrhage (EIPH). In: CONGRESS ON EQUINE MEDICINE AND SURGERY, 11., 2009. Geneva. Proceedings... Geneva: [s.n.], 2009. p.77-82.
MICHELOTTO JÚNIOR, P.V.; BIAVA, J.S.; GONÇALVES, R.C. et al. Aspirado traqueal de cavalos clinicamente sadios da raça Quarto de milha após prova de três tambores. Arch. Vet. Sci., v.12, p.1-7, 2007.

POOLE, D.C.; ERICKSON, H.H. Heart and vessels: function during exercise and response to training. In: HINCHCLIFF, K.W., KANEPS, A.J., GEOR, R.J. (Eds.). Equine sports medicine and surgery. Philadelplia: W.B. SAUNDERS, 2004. p.699-727.

RAKER, C.; BOLES, C. Pharyngeal lymphoid hyperplasia in the horse. J. Equine Med. Surg., v.2, p.202-207, 1978.

SANCHEZ, A.; COUETIL, L.L.; WARD, M.P.; CLARK, S.P. Effect of airway disease on blood gas exchange in racehorse. J. Vet. Intern. Med., v.19, p.87-92, 2005.

SILVA, M.A.G.; GOMIDE, L.M.W.; DIAS, D.P.M. et al. Equilíbrio ácido-base em equinos da raça Quarto de milha participantes da prova dos Três Tambores. Rev. Bras. Med. Vet., v.35. p.188-192, 2013.

SILVA, M.A.G.; MARTINS, C.B.; GOMIDE, L.M.W. et al. Determinação de eletrólitos, gases sanguíneos, osmolalidade, hematócrito, hemoglobina, base titulável e anion gap no sangue venoso de equinos destreinados submetidos a exercício máximo e submáximo em esteira rolante. Arq. Bras. Med. Vet. Zootec., v.61, p.1021-1027, 2009.

THOMAS, D.P.; FREGIN, G.F.; GERBER, N.H.; AILES, N.B. Effects of training on cardiorespiratory function in the horse. Am. J. Physiol., v.245, p.160-165, 1983.

WILKINS, P.A.; GLEED, R.D.; KRIVITSKI, N.M.; DOBSON, A. Extravascular lung water in the exercising horse. J. Appl. Physiol., v.91, p.2442-2450, 2001 